\title{
The effect of fructose on NAFLD in children and adolescents: a narrative review
}

\author{
Abstract \\ Background: Fructose is used in commercial products, sweets, and soft drinks. Several \\ studies indicate that the consumption of this processed fructose is related to NAFLD. \\ The purpose of this narrative review is to examine this issue in children, according to the \\ bibliography.
}

Methods: We conducted a bibliographic search in the PubMed-Medline database where we searched for papers published from 2015 to 2018

Results: 4 recent studies we found in the literature, they had a similar population ( 7 to 18 years old \& NAFLD diagnosis), followed several different methods of methodology, three of which were interventional researches, and one was observable. All had the same final conclusion and agrees that consumption of dietary fructose acts on the pathophysiology of NAFLD with a negative impact.

Conclusion: These data support the idea for a general intervention focusing on limiting fructose as a way of reducing the percentage of children with NAFLD.
Volume 8 Issue 2 - 2018

\author{
Chatzipapa N,' Kolanis S2 \\ 'Department of Pediatrics, AUTH School of Medicine, Greece \\ ${ }^{2}$ Medical Research Methodology,AUTH School of Medicine, \\ Greece
}

Correspondence: Chatzipapa Nikoleta, Department of Pediatrics, AUTH School of Medicine, Ippokrateio GH, Greece, Email nikoletaxatzhpapa@hotmail.com

Received: April 07, 2018 | Published: April 18, 2018

Keywords: fatty liver, fructose, children, adolescents

Abbreviations: NAFLD, non-alcoholic fatty liver disease is fatty liver; FFQ, food frequency questionnaire; MRI, Magnetic resonance imaging

\section{Introduction}

Lipid infiltration in hepatic parenchyma cells leads to abnormal accumulation of lipids, usually in the form of triglycerides (either as a single large droplet or with multiple small droplets). The fatty liver caused by an imbalance in the metabolism of fatty acids. ${ }^{1}$ The nonalcoholic fatty liver disease is fatty liver (NAFLD) without excessive alcohol consumption. ${ }^{2}$ NAFLD includes a range of pathological conditions from simple steatosis to non-alcoholic steatohepatitis, fibrosis, cirrhosis and hepatocellular carcinoma. The prevalence of the disease in Europe and the US approaching 20-30\%. ${ }^{1}$ It is now the most common form of the chronic liver disease in childhood and adolescence, affecting about $10-20 \%$ of the general pediatric population. In the next 10 years, NAFLD in pediatric patients is expected to become the most common cause of liver disease, hepatic insufficiency, and cause of liver transplantation in the western world. The liver is responsible for many metabolic and anabolic processes that take place in various anatomical compartments of the organ. Imbalance in fatty acid synthesis, beta-oxidation, and triglyceride accumulation lead to fat accumulation in hepatocytes. Accumulation of topic fat, mainly triglycerides, leads to simple steatosis. The most common cause of fatty liver can be attributed to increased caloric intake, which exceeds the caloric needs of the body. And while simple steatosis is considered to be a relatively benign liver disease, clinically it seems that $15-25 \%$ of patients could develop NAFLD. Also, some of the patients develop long-term fibrosis, cirrhosis or even hepatocellular carcinoma. Risk factors for the development of NAFLD are low physical activity, eating habits, intestinal microbial, genetic and regulatory factors and oxidative stress. ${ }^{1}$

Fructose is naturally found in fruits, honey, and vegetables. However, it is used very much in commercial products, sweets, and soft drinks, mainly in the form of corn syrup with a high concentration of fructose. Several studies show that the consumption of this processed fructose is also related to NAFLD. ${ }^{1}$ Several hypotheses correlate the high fructose consumption with abnormalities in liver structure and function, insulin resistance and microbial flora and a direct effect of fructose on the pathogenesis of NAFLD. ${ }^{3}$

Fructose is effectively converted to glyceraldehyde 3-phosphate, however, avoiding controlling the rate of glucose metabolism in hepatocytes. A diet rich in fructose promotes biogenesis "de novo" and mitigates the effect of liver enzymes of lipogenesis. These conditions lead the liver to inflammatory processes. ${ }^{4}$ Also increases mitochondrial coupling leading to mitochondrial stress. Another important point is that high sugar and fat diets cause fructokinase associated to steatohepatitis, indicating a key role for fructose (derived from sucrose) in this development. But it is not only dietary fructose which causes metabolic imbalance, also the production of endogenous fructose and its metabolism in the liver, which was found to contribute to the growth of NAFLD according to some researchers. ${ }^{1}$ The purpose of our narrative review is to check whether there is a correlation to the pathogenesis of NAFLD and the increased consumption of fructose in children, according to the bibliography.

\section{Methodology}

We conducted a bibliographic search in the PubMed-Medline database where we searched for clinical researches and reviews published from 2015 to 2018 referring to children between the ages of 6-18 with NAFLD. The PubMed Search Strategy: [(Non-alcoholic fatty liver disease) AND fructose consumption]. The papers we chose to include in our research, more detailed, had the following characteristics: Age from 7 to 18 years, NAFLD diagnosis based on international criteria (with histological confirmation and proper imaging examinations), dietary interventions or weighted-certified questionnaires, healthy control group as a measure of intervention or lifestyle. We excluded papers on which NAFLD was the result of another chronic disease, cancer or metastatic disease, alcohol consumption. 


\section{Results}

Schwarz et al. ${ }^{3}$ included 41 non-diabetic obese children (9-18 years old) and metabolic syndrome based on food frequency questionnaires (FFQ) found to be consumers with a high sugar content $(>15 \%$ sugar, $>5 \%$ fructose) and age average of 13 years old. Children were given all meals for 9 days with the same energy and macronutrient composition as their usual diet but with sugar-substituted starch, with fructose at day 0 being $12-18 \%$ depending on the indicated intake which everyone had, reaching $4 \%$ on the 10 th day. Before and after the restriction of fructose were conducted measurements of liver fat, the subcutaneous fat and visceral fat in MRI, and glucose curve. At the beginning of the study, 25 participants had increased liver fat (5\% fat fraction) and 15 low liver fat. From day 0 to day 10, liver fat decreased by a median of $7.1 \%$ to $3.8 \%(\mathrm{P}<.001)$. Visceral adipose tissue decreased from $123 \mathrm{~cm}^{3}$ to $110 \mathrm{~cm}^{3}(\mathrm{P}<.001)$, while subcutaneous adipose tissue did not change much.

The findings of this research show that even the short-term (9-day) limited diet in fructose reduces fat in the liver, visceral adipose tissue and as a secondary effect of improving the insulin curve in obesity children. Fructose consumption and hyperinsulinemia are important determinants of fatty liver production.

Mager et al. ${ }^{2}$ hypothesized that diet with a decrease in fructose, glycemic index, and glycemic load for more than 6 months will lead to improvements in body composition, liver dysfunction and cardiometabolic risk in obese children with NAFLD. For this purpose, 12 (obese and non-obese) children with a clinical diagnosis of NAFLD and 14 normal healthy children were examined as a control group (7-18 years) for a period of 6 months. Before the start of the program and after 6 months, anthropometric data, laboratory tests, and pressure measurements were obtained, except for liver ultrasound and liver biopsy where clinical evidence was available. Dietary intake was evaluated at each visit (3 and 6 months) with a 3- day dietary record. Children and their families received an indicative menu that they should follow in the next 6 months. Significant reductions in systolic blood pressure (SBP) were found in the analysis of the results, while the decrease in dietary intake of fructose and the glycemic load was also associated with a decrease in body fat $(\mathrm{P}=.04)$. The largest changes in the first 3 months of intervention occurred in children with NAFLD when their dietary intake was like that of the control group. And the present study has shown that changes in the quality of carbohydrate intake (such as a decrease in total fructose uptake) are associated with decreases in cardiovascular risk factors and hepatic dysfunction in obese children with NAFLD.

Sullivan et al. ${ }^{5}$ focused on the measurement of fructose absorption and metabolism in children with NAFLD compared to obese children and normal weight children in control groups, a total of 24 children took part in the study (9 NAFLD, 6 obese and 9 normal weight, 8-18 years old). Participants were asked to refrain from food and beverages containing sugar for 24 hours before the consumption of drinking fructose. After 8 hours of fasting, the children drink oral fructose ( $1 \mathrm{~g} / \mathrm{kg}$ ideal weight) dissolved in $8 \mathrm{oz}$ of water, followed by 6 hours of fasting. It was obtained from blood samples (for glucose, triglycerides, free fatty acids, uric acid and fructose concentrations) before consumption of fructose and at 30, 60, 90, 120, 180, 270 and 360 minutes after consumption of fructose. Insulin count was also measured at 30,60,90, 120 minutes, and morning urea before consumption of fructose.
After fructose consumption, children with NAFLD compared to normal control group children had increased serum glucose, insulin and uric acid $(p<0.05)$ but lower fructose excretion $(p=0.002)$. Similarly, children with NAFLD on obese children in the control group had similar serum glucose after consumption of fructose, insulin, uric acid, but increased fructose excretion $(p=0.02)$. From the above, the researchers concluded that children with NAFLD absorb and metabolize fructose more effectively than non-afflicted children, and this is related to the deterioration of the metabolic profile following consumption of fructose, contributing to the pathophysiology of the disease. In another study Mosca et al. ${ }^{4}$ researchers decided to investigate as factors related to NAFLD in children and adolescents uric acid concentrations and consumption of fructose are independently related to the disease. Also, because fructose consumption may increase uric acid concentrations, they examined whether fructose consumption was independently correlated with uric acid concentrations in this population. 271 obese teenagers with NAFLD (mean age 12.5 years old) included in the study. Uric acid was measured in children and FFQ was used to estimate the daily intake of all dietary ingredients for each patient and using a daily food intake formulation using fructose intake.

Statistical analysis of the data showed that hyperuricemia was present in $47 \%$ of patients. Also, both uric acid $(p=0.004)$ and fructose consumption $(\mathrm{p}=0.001)$ were independently associated with NAFLD, while fructose consumption was independent of hyperuricemia $(p=0.01)$. The present study, therefore, shows that in children and adolescents with a histological diagnosis of NAFLD, uric acid concentrations and consumption of dietary fructose are independent and positively associated with the disease. The data also show that dietary fructose consumption is positively and independently associated with hyperuricemia.

\section{Discussion}

Over the past two decades, the prevalence of NAFLD has doubled in adolescents and adults, with the current United States rate estimated at $50 \%$. It is very important to understand the risk factors for this disease in children and adolescents to design effective interventions that can be used safely for the treatment of this new group of patients. Studies in obese children showed improvements in liver histology after weight loss, and recent data suggest a correlation of increased consumption of sugar-containing beverages containing fructose and glucose as sucrose disaccharide or sugar. Increased intake of fructose from the diet can be important in the pathogenesis of NAFLD. This is confirmed by the review of the recent literature, whose findings we presented in the Results. The studies presented all concern the same population, children, and adolescents with NAFLD, and 3 of the 4 studies included obese children suffering from the disease. Analytical characteristics of the population are summarized in Table 1.

To our great surprise, we discovered that 4 recent studies we found in the literature, although they had a similar population, followed several different methods of methodology, three of which were interventional researches, and one was observable. Table 2 summarizes the methods used. However, in spite of the different methodology followed, the final conclusion was the same and agrees that consumption of dietary fructose acts on the pathophysiology of NAFLD with a negative impact, whereas in one of them it was found to act even as an independent agent. ${ }^{4}$ 
Table I Overall characteristic of participants

\begin{tabular}{lllll}
\hline Data/Article & $\begin{array}{l}\text { Schwarz } \\
\text { et al. }{ }^{3}\end{array}$ & $\begin{array}{l}\text { Mager } \\
\text { et al. }{ }^{2}\end{array}$ & $\begin{array}{l}\text { Sullivan } \\
\text { et al. }{ }^{5}\end{array}$ & $\begin{array}{l}\text { Mosca } \\
\text { et al. }{ }^{4}\end{array}$ \\
\hline $\begin{array}{l}\text { Age (years) median } \\
\text { Participants }\end{array}$ & 13 & 13,3 & 13,7 & 12,5 \\
Boys & 41 & 26 & 24 & $27 \mid$ \\
Girls & 15 & 16 & 12 & 155 \\
Children with NAFLD & 0 & 10 & 12 & 116 \\
$\begin{array}{l}\text { Obese children with } \\
\text { NAFLD }\end{array}$ & 41 & 12 & 9 & $27 \mid$ \\
\hline
\end{tabular}

Table 2 All methods used in studies

\begin{tabular}{lllll}
\hline Data/Article & $\begin{array}{l}\text { Schwarz } \\
\text { et al. }{ }^{3}\end{array}$ & $\begin{array}{l}\text { Mager } \\
\text { et al. }{ }^{2}\end{array}$ & $\begin{array}{l}\text { Sullivan } \\
\text { et al. }{ }^{5}\end{array}$ & $\begin{array}{l}\text { Mosca } \\
\text { et al. }{ }^{4}\end{array}$ \\
\hline $\begin{array}{l}\text { Obese control group } \\
\begin{array}{l}\text { Normal weight control } \\
\text { group }\end{array}\end{array}$ & 0 & 0 & 6 & 0 \\
$\begin{array}{l}\text { Without control group } \\
\text { Diet program }\end{array}$ & Yes & Yes & No & No \\
Oral fructose & Yes & Yes & No & No \\
FFQ & No & No & Yes & No \\
3- day dietary record & No & Yes & No & No
\end{tabular}

We also found a great deal of interest in a study, ${ }^{3}$ which showed that even with a short-term change in quality carbohydrate intake, positive results could be seen in suffering children, which allowed us to consider the benefits of a long-term change in their diet. Very important are the findings of another research, ${ }^{5}$ which focused on the direct metabolism of fructose, concluding that the diseased children showed different and better metabolism of fructose as reported in relation to healthy children in the control group. At this point, it is necessary to mention again that the fructose to which we refer concerns added sugars that vary in the Western diet and not the natural sugars found in the Mediterranean diet. It is emphasized that great emphasis should be placed on the quality of the carbohydrate consumed, which was also shown in the study, ${ }^{2}$ presented above. Finally, it is worth mentioning that 2 of the studies we chose to include this review had secondary results. It has been found that as well as to fructose consumption, hyperinsulinemia is a determinant of fatty liver production, with an association of consumption of added sugars and hyperinsulinemia. Also, the role of uric acid was also examined, and consumption of fructose seemed positive and independent of hyperuricemia, as reported. The above secondary results of studies reinforce the incrimination of excessive consumption of fructose both as a direct and indirect agent.

These data support the idea for a general intervention focusing on limiting fructose as a way of reducing the percentage of children with NAFLD. Further research may shed light on the determination of the efficacy of long-term fructose restriction as a means of preventing or treating NAFLD and its associated metabolic lesions.

In addition, it is necessary to mention the urgent need for public health and the concern to reduce the consumption of sugar as a mean of improving the health of children and the general population.

\section{Acknowledgment \\ None. \\ Conflict of interest}

There is no conflict of interest between researchers as work was done for academic purposes and is the product of an independent research.

\section{References}

1. Alwahsh SM, Gebhardt R. Dietary fructose as a risk factor for nonalcoholic fatty liver disease (NAFLD). Arch Toxicol. 2017;91:15451563 .

2. Mager DR, Iñiguez IR, Gilmour S, et al. The Effect of a Low Fructose and Low Glycemic Index / Load (FRAGILE ) Dietary Intervention on Indices of Liver Function, Cardiometabolic Risk Factors, and Body Composition in Children and Adolescents With Nonalcoholic Fatty Liver Disease ( NAFLD ). JPEN J Parenter Enteral Nutr. 2015;39(1):73-84.

3. Schwarz JM, Noworolski SM, Erkin-Cakmak A, et al. Effects of Dietary Fructose Restriction on Liver Fat, De Novo Lipogenesis, and Insulin Kinetics in Children with Obesity. Gastroenterology. 2017;153:743-752.

4. Mosca A, Nobili V, De Vito R, et al. Serum uric acid concentrations and fructose consumption are independently associated with NASH in children and adolescents. J Hepatol. 2017;66:1031-1036.

5. Sullivan JS, Le MT, Pan Z, et al. Oral fructose absorption in obese children with non-alcoholic fatty liver disease. Pediatr Obes. 2015;10(3):188-195. 